\title{
Stroke care: the way forward
}

T W J Watson, J E Simon, A M Buchan

\section{"Nothing will ever be attempted if all possible objections must first be overcome" (Samuel Johnson)}

The development of coronary care units, cardiac rehabilitation programmes, and thrombolysis revolutionised the management of acute myocardial infarction. Similarly, the development of stroke wards and stroke teams, thrombolysis, and aggressive early rehabilitation have revolutionised stroke care. Unfortunately acceptance and translation of these concepts into clinical practice has been slow. It is imperative that resources are committed to making this new standard of stroke care widely available.

\section{THE EVIDENCE FOR THROMBOLYSIS}

In 1995 the National Institute of Neurological Disorders and Stroke (NINDS) rt-PA stroke study demonstrated the efficacy of recombinant tissue type plasminogen activator (rt-PA) for the treatment of acute ischaemic stroke (AIS) when administered within three hours of symptom onset. ${ }^{1}$ This randomised controlled trial of 624 patients reported a $13 \%$ absolute increase in favourable outcome at three months (defined as a modified Rankin Scale score (mRS) $0-1)$. The number of patients needed to treat (NNT) to result in one additional favourable outcome over placebo was seven. rt-PA was associated with an increased absolute risk of symptomatic intracerebral haemorrhage ( $\mathrm{SICH})(6 \% v$ $1 \%$ for placebo) but there was no significant increase in mortality. Intravenous rt-PA was approved for use in stroke by the Food and Drug Administration in the United States the following year. In 1999 the Therapeutics Product Programme in Canada provisionally approved the use of rt-PA on the condition that a registry of treated cases was maintained. ${ }^{2}$ This was the first licensed treatment for AIS and was embraced with enthusiastic optimism by a number of stroke centres, particularly those who had direct experience with rt-PA in the NINDS trial. In September 2002 the European Agency for the Evaluation of Medicinal Products licensed rt-PA on the proviso that a further randomised trial be conducted for treatment three to four hours postsymptom onset (ECASS III) and a European registry is maintained (Safe Implementation of Thrombolysis in Stroke
Monitoring System, SITS-MOST; www.emea.eu.int/pdfs/human/referral/ 334602en.pdf).

Meta-analysis of the current evidence for thrombolysis supports the findings of the NINDS study, ${ }^{3}$ however the recanalisation rate after intravenous therapy for proximal middle cerebral artery occlusions is probably only $30 \%{ }^{4}$ In an attempt to improve on this recanalisation rate and extend the time window for therapy, trials of intra-arterial (IA) thrombolysis were undertaken. PROACT II was a randomised controlled trial of IA pro-urokinase in 180 patients with angiographically proven middle cerebral artery occlusion and severe stroke (average National Institute of Stroke Scale score of 17). ${ }^{5}$ In spite of a mean time of 5.3 hours from stroke onset to initiation of IA pro-urokinase, this trial demonstrated a $15 \%$ absolute increase in favourable outcomes in the treatment group (mRS 0-2). There was an increased risk of SICH $(10 \% \vee 2 \%)$ but no increase in mortality. A number of case series of IA and combined IV and IA rt-PA followed and recent meta-analysis of IA thrombolysis suggests an 18.5\% absolute and $80 \%$ relative increase in favorable outcomes, NNT of six and reduced mortality in the IA thrombolysis group with initiation of therapy up to six hours from symptom onset. ${ }^{6}$

To put this in perspective, preventive therapies such as aspirin administered following stroke provide an absolute risk reduction (ARR) of $0.9 \%$ for early recurrence or death with NNT of $111 .^{7}$ Warfarin in older patients with uncomplicated atrial fibrillation offers an ARR of $1.5 \%$ per year compared with aspirin. ${ }^{8}$ The benefits and risks of rt-PA are very similar to carotid endarterectomy for symptomatic high grade carotid stenosis ( $>$ $70 \%)$ in which the North American Symptomatic Carotid Endarterectomy Trial (NASCET) demonstrated a $17 \%$ ARR of ipsilateral stroke over two years, with a NNT of six despite a 5.6\% perioperative risk of stroke or death. ${ }^{9}$ Like surgery and warfarin, rt-PA is potentially dangerous. However, there is compelling evidence that when used in accordance with present guidelines, under the direction of an experienced stroke team, rt-PA is a powerful and relatively safe therapeutic agent whose impact on acute ischaemic stroke can be dramatic. A number of Canadian, American, and European stroke centers are now thrombolysing in excess of $10 \%$ of all ischaemic strokes.

\section{WHAT HAVE WE LEARNED?}

A number of conclusions can be drawn from the past decade of stroke research. First and foremost, in AIS with demonstrated arterial occlusion the only evidence-based approach to improve outcome acutely is disruption of thrombus and reperfusion of the zone of ischaemia.

The overriding priority remains patient safety, but with AIS the order of the day is urgency in administering the thrombolytic. The goal is not just treatment in under three hours but treatment as soon as possible. There is a significant decline in the probability of favourable outcome for every additional 10-15 minutes delay in the initiation of reperfusion therapy. ${ }^{10}$ This urgent time frame for investigation and treatment has had the beneficial spin-off of producing a wealth of new information regarding the evolution of AIS.

Multidisciplinary acute stroke teams can be developed and organised to function quickly and efficiently. ${ }^{11}$ Fifty percent of the patients in the NINDS trial were assessed clinically, CT scanned, randomised, and had treatment started within 90 minutes of symptom onset. In experienced stroke centres door to needle times of 30 minutes are becoming routine in uncomplicated cases and these results are being achieved in public healthcare systems, such as exists in Canada. This requires recruitment, training, and goal directed restructuring of existing resources and personnel.

One size does not fit all. Optimal stroke therapy must be tailored to specific stroke subtypes. Rapid identification of the mechanism of stroke, the site of vascular involvement, and the assessment of tissue viability requires appropriate resources and organisation to conduct CT and/or MR imaging on an emergency basis. Twenty four hour, seven days a week availability of emergency CT, and expertise in neuroradiology and interventional neuroradiology are essential. Increasingly sophisticated imaging techniques are being developed to help guide decision making on the basis of a "tissue window" and extend treatment options beyond the current "time window". ${ }^{12-13}$

All stroke patients benefit from urgent investigation and initiation of treatment, not just those receiving rt-PA. Evidence indicates that care on a stroke ward, under the direction of a stroke team with early and aggressive rehabilitation, improves outcome of all forms of stroke whether they are rt-PA candidates or not. ${ }^{14}$ 


\section{Keypoints: essential components for acute stroke care}

- Rapid triage of patients and public education to recognise stroke symptoms

- Comprehensive stroke team and dedicated stroke unit: experienced stroke physician, stroke nurse coordinator, neuroradiologist, rehabilitation specialist, physiotherapist, occupational therapist, speech and language therapist, and dietician

- Rapid access to CT (and MRI)

- Ability to deliver IV rt-PA 24 hours a day, 7 days a week

- Access to a monitored bed with one-to-one nursing

- Access to IA/interventional approaches

\section{A CALL FOR CHANGE}

"Much has been done but when we look ahead at what remains we see only a beginning has been made" (Sir Wm. Osler, 1907). Thrombolytic therapy has sparked a revolution in acute stroke care but rt-PA is not the final answer. rt-PA will eventually be replaced by combinations of pharmacological and mechanical techniques of thrombolysis with improved efficacy and risk/benefit ratio. The presently limited time window for reperfusion therapy will also be extended by combinations of neuroprotective agents and interventions such as hypothermia, increasing the number of patients that can be thrombolysed. Numerous questions remain but in the clinical arena the principle task is to close the gap between what is already known and what is being translated into clinical practice.

Stroke is the leading cause of long term disability in the adult population in Britain, with stroke patients occupying $20 \%$ of hospital beds. ${ }^{15}$ It is time to give the same priority to the training of multidisciplinary stroke teams and development of stroke units as was given to coronary care units and the management of myocardial infarction. Governments, health trusts, and funding agencies need to made aware of the critical dependence of modern stroke care on these elements and on emergency access to CT and MR scanning. The potential savings, from reductions in the number of patients requiring lengthy hospitalisations and long term care, quickly offset the capital costs. ${ }^{16-17}$

\section{CONCLUSION}

The call for a change in British stroke care is growing ${ }^{18-19}$ and what is needed now is a National Service Framework for Stroke (see www.doh.gov.uk/nsf). Debate over evidence of the efficacy of rt-PA should not distract us. The critical issue is to create the necessary infrastructure of acute stroke teams, stroke units, and neuroradiology facilities to deliver a new standard of stroke care. Acute stroke treatments will continue to be improved and refined, but without organised acute stroke care, patients in Britain will be denied benefit from these developments.

I Neurol Neurosurg Psychiatry

2003;74:411-412

\section{Authors' affiliations}

T W J Watson, J E Simon, A M Buchan, University of Calgary

For correspondence: T W J Watson; watsont@ucalgary.ca

\section{REFERENCES}

1 The National Institute of Neurological Disorders and Stroke rt-PA stroke study group. Tissue plasminogen activator for acute ischemic stroke. N Engl J Med 1995;333:1581-7.

2 Hill MD, Buchan AM. Methodology for the Canadian Activase for Stroke Effectiveness Study (CASES). CASES Investigators. Can J Neurol Sci 2001;28:232-8.
3 Wardlaw JM, del Zoppo G, Yamaguchi T. Thrombolysis for acute ischaemic stroke. Cochrane Database Syst Rev 2000;2:CD0002 13.

4 del Zoppo GJ, Poeck K, Pessin MS, et al. Recombinant tissue plasminogen activator in acute thrombotic and embolic stroke. Ann Neurol 1992:32:78-86.

5 Furlan A, Higashida R, Wechsler L, et al. Intra-arterial prourokinase for acute ischaemic stroke - The PROACT II study: A randomized controlled trial. JAMA 1999;282:2003-11.

6 Lisboa RC, Jovanovic BD, Alberts M. Analysis of the safety and efficacy of intra-arterial thrombolytic therapy in ischemic intra-arterial thrombolytic therapy in
stroke. Stroke 2002;33:2866-71

7 Chen ZM, Sandercock P, Pan HC, et al, on behalf of the CAST and IST collaborative groups. Indications for early aspirin use in acute ischaemic stroke: a combined analysis of 40,000 randomised patients from the Chinese acute stroke trial and the international stroke trial. Stroke 2000;31:1240-9.

8 Segal JB, McNamara RL, Miller MR, et al. Anticoagulants or antiplatlet therapy for non-rheumatic atrial fibrillation and flutter. Cochrane Database Syst Rev 2001;1:CD001938.

9 North American Symptomatic Carotid Endarterectomy Trial Collaborators. Beneficial effect of carotid endarterectomy in symptomatic patients with high grade stenosis. NEJM 1991;325:445-53

10 Marler JR, Tilley BC, Lu M, et al. Early stroke treatment associated with better outcome: the NINDS rt-PA stroke study. Neurology 2000;55: 1649-55.

11 Hill MD, Barber PA, Demchuk AM, et al. Building a "brain attack" team to administe thrombolytic therapy for acute ischemic stroke. Can Med Assoc J 2000;162: 1589-93.

12 Albers GW. Expanding the window for thrombolytic therapy in acute stroke. The potential role of acute MRI for patient potential role of acute MRI for patient
selection. Stroke 1999;30:2230-7.

13 Wintermark M, Bogousslavsky J. Imaging of acute ischemic brain injury: the return of computed tomography. Curr Opin Neurol 2003:16:59-63.

14 Organised inpatient (stroke unit) care for stroke. Stroke Unit Trialists' Collaboration. Cochrane Database Syst Rev 2002;1:CD000197.

15 Wade DT. Stroke /acute cerebrovascular disease). Health care needs assessment. Vol 1. Oxford: Radcliffe Medical Press, 1994:111-255.

16 Gleason S, Furie KL, Lev MH, et al. Potential influence of acute CT on inpatient costs in patients with ischemic stroke. Acad Radiol 2001;8:955-64.

17 Fagan SC, Morgenstern LB, Petitta A, et al. Cost-effectiveness of tissue plasminogen activator for acute ischemic stroke. Neurology 1998;50:883-90.

18 Lindley RI. Thrombolysis for acute stroke in the United Kingdom. Age and Ageing 2002;31-S3:28-30.

19 Warlow C, Wardlaw J. Therapeutic thrombolysis for acute ischaemic stroke. BM 2003;326:233-4. 


\section{Visible infarction on CT after stroke-what does it tell?}

\section{R Kay, A C F Hui}

logistic reasons why CT remains the imaging modality of first choice. ${ }^{3}$

What, then, is the value of CT when infarction is not seen? Most importantly, it is more reliable than MR in ruling out cerebral haemorrhage. It may even predict a better prognosis, although much depends on other factors. If the CT is performed within 3 hours of stroke, it helps to identify patients suitable for thrombolytic therapy, ${ }^{4}$ provided that it is read by a specialist. Perhaps the biggest drawback of a "negative CT" is that it cannot confirm that any infarction has taken place. This makes giving potentially harmful therapies rather awkward, and does little to reassure the patient's family.

J Neurol Neurosurg Psychiatry 2003;74:413 haematoma spring into mind. Not all infarcts are visible on $\mathrm{CT}$, especially in the first few hours. In the paper by Wardlaw et al on p 452-458, ${ }^{1}$ among 12550 patients who were enrolled in the International Stroke Study (1991-96) only $50 \%$ had visible infarction, even up to 48 hours after stroke.

Compared with patients without visible infarction, those who had were $25 \%$ more likely to have a poor outcome (defined as death or dependency) at six months. There was, however, neither an association between visible infarction and subsequent haemorrhagic transformation of the infarct (which occurred in less than $1 \%$ of all patients), nor any interaction between visible infarction and treatment allocation (aspirin or heparin) with the six month outcome.

As functional outcome reflects the amount of brain infarcted, it can be expected that early visualisation of this process will lead to a poorer outcome. On the other hand, haemorrhagic transformation of the infarct is a multifactorial process dependent not only on the size of the infarct, but also on the blood pressure, blood coagulability, and a host of other factors such as whether and when reperfusion has occurred. Thus, early visualisation of infarction may not independently predict haemorrhagic transformation.

Ever since the late 1990s, when thrombolytic treatment gained approval from the US Food and Drug Administration for the treatment of cerebral infarction within 3 hours of onset, there has been intense interest in visualising infarction as early as possible. A variety of "early infarct signs" on CT, such as loss of cortical grey-white matter differentiation and loss of the insular ribbon, have been proposed, ${ }^{2}$ but these signs are generally subtle and lacking in interrater agreement. Posterior fossa strokes are also not included in such schemes. Newer techniques (diffusion weighted imaging and perfusion imaging) using MR are much more sensitive but are not as widely available as CT. Even in centres where MR is available, there are many

\section{Authors' affiliations}

R Kay, A C F Hui, Department of Medicine and Therapeutics, Prince of Wales Hospital, Shatin, Hong Kong

Correspondence to: Professor R Kay; Lichikay@cuhk.edu.hk

\section{REFERENCES}

1 Wardlaw JM, West TM, Sandercock PAG, et al. Visible infarction on CT is an independent predictor of poor functional outcome after stroke, and not of haemorrhagic

transformation. J Neurol Neurosurg Psychiatry 2003;74:452-8.

2 Jager HR. Diagnosis of stroke with advanced CT and MR imaging. Br Medical Bull 2000;56:318-33.

3 Tatlisumak T. Is CT or MRI the method of choice for imaging patients with acute stroke? Why should men divide if fate has united? Stroke 2002;33:2144-5.

4 Brott T, Bogousslavsky J. Treatment of acute ischemic stroke. N Engl J Med 2000;343:710-22.

\section{Current research on diagnosing dementia}

\section{J Warner}

\section{Quis custodiet ipsos Custodes}

Q uis custodiet ipsos Custodes (who is to guard the guards themselves?). ${ }^{1}$ What is the link between this rather pithy observation by a first century AD Roman poet and current research on diagnosing dementia? Read on!

The paper by Tian et al (this issue, pp $433-438)^{2}$ explores the clinical utility of predictive testing of individuals with early cognitive problems. The hinterland between normal cognition and dementia is a nosological and terminological minefield. The problem is that before people develop full-blown dementia, they often pass through a stage of "pre-dementia", referred to in Tian et al's article as "questionable dementia". Mild cognitive impairment, benign senescent forgetfulness, and age associated memory impairment are other, subtly different reworkings of the same phenomenon. In Tian et al's paper, questionable dementia is defined as either the presence of subjective memory impairment affecting social or occupational functioning, but in the absence of impairment in other facets of cognition; or cognitive deficits in one or more domains without impairment of functioning. However, not everyone with the pre-dementia syndromes will go on to develop full-blown dementia, nor are all cases of dementia presaged by this phase.

Accurate early diagnosis of dementia is becoming increasingly important. In people with established dementia, early treatment with cholinesterase inhibitors may confer greater benefit than delayed treatment ${ }^{3}$ and provides the patient and family with a longer lucid time to make 
plans, do the undone, discuss treatments, and come to terms with the diagnosis.

The most useful clinical measure for patients is the positive predictive value (PPV). This answers the question "If I test positive, what is the likelihood that I will develop (or have) the condition the test is testing for?" The problem is that as the PPV rises, the negative predictive value (If the test is negative, what is the likelihood I will not have the disease) falls. In this case, Tian and co-workers found PPVs around $85 \%$ and negative predictive values around $45 \%$. As they point out in their shrewd discussion, these figures are not helpful for most patients, who prefer to deal in certainties.

A significant issue with all research in this area is having a robust reference (gold) standard to diagnose dementia and evaluate the usefulness of your screening test. Most researchers rely on old favourites such as the DSM or NINCDS, although clinicians may be less rigid. When calculating parameters such as sensitivity and PPV, one has to regard these gold standards as inviolable. They are not. In fact, the sensitivity of antemortem diagnosis using these instruments in centres of excellence is at best around $90 \%$ when compared with the next hierarchy; histological examination. ${ }^{4}$ But who is to say that histology is always accurate (it cannot be, no test is). One can rapidly descend into a mire of iterative complexity and nosological uncertainty. The parallels between reference diagnostic standards and the Roman guard become obvious and we are left having to trust what we have, but feeling a little uneasy about it.

J Neurol Neurosurg Psychiatry 2003;74:413-414

\section{Author's affiliation \\ J Warner, Imperial College School of Medicine, Paterson Centre, 20 South Wharf Road, London W2 IPD \\ Correspondence to: J Warner; i.warner@ic.ac.uk}

\section{REFERENCES}

1 Cohen JM, Cohen M (eds). Juvenal Satires vi 347. The Penguin dictionary of quotations. London: Omega Books, 1986:214.

2 Tian J, Buck RS, Haworth J, et al. Neuropsychological prediction of coversion to dementia from questionable dementia: statistically significant but not yet clinically useful. I Neurol Neurosurg Psychiatry 2003;74:433-8

3 Doraiswamy PM, Krishnan KR Anand R, et al. Long-term effects of rivastigmine in moderately severe Alzheimer's disease. Does early initiation of therapy offer sustained benefits? Prog Neuro Psychopharmacol Biol Psychiatry 2002;26:705-12.

4 Burns A, Luthert P, Levy R, et al. Accuracy of clinical diagnosis of Alzheimer's disease. BM 1990;301:1206. 